\title{
Arterial microembolisation: an unusual presentation of dilated cardiomyopathy
}

\author{
Robert L Gillespie, G Martin Mullen, Maria Rosa Costanzo-Nordin
}

\begin{abstract}
Systemic embolisation is common in patients with dilated cardiomyopathy. Microembolisation as a presenting sign of dilated cardiomyopathy, however, has not been reported before. A 37 year old woman in whom dilated cardiomyopathy presented as arterial microembolisation to the toes is described.
\end{abstract}

Dilated cardiomyopathy is a clinical syndrome characterised by ventricular dilatation and associated systolic dysfunction in the absence of pericardial, valvar, or coronary artery disease. ${ }^{1}$ The clinical presentation, course, and prognosis of dilated cardiomyopathy are highly variable. In most studies of the clinical presentation of dilated cardiomyopathy, embolism was identified as a frequent and serious complication of this disease. ${ }^{2}$ It is estimated that at least $11 \%$ of patients with dilated cardiomyopathy have one or more embolic events during the course of their illness. ${ }^{3}$ The timing and severity of thromboembolism in patients with dilated cardiomyopathy are highly variable. In some instances embolic events are clinically silent and are detected only at necropsy as peripheral infarcts in patients dying of progressive congestive heart failure. More commonly, embolism occurs long after the onset of clinical evidence of congestive heart failure. ${ }^{4}$ In a few patients embolic events precede all other cardiac symptoms and the clinical effects of peripheral infarcts may overshadow the primary cardiac disease and confuse the diagnosis. Retrospective evaluation of these patients, however, has generally shown that radiographic cardiomegaly or electrocardiographic abnormalities were present at the time of embolisation. ${ }^{45}$

The systemic emboli described in patients with dilated cardiomyopathy are generally large and affect, in order of decreasing frequency, the lungs, the kidneys, the spleen, and the brain. ${ }^{6-10}$ The occurrence of arterial microemboli as a presenting feature of dilated cardiomyopathy has not been reported before. We describe a patient in whom arterial microembolisation was the presenting sign of dilated cardiomyopathy.

\section{Case report}

A 37 year old white woman was admitted to the hospital with severe pain and discoloration in both fifth toes. She had been in good health until a month before admission when she noticed a painless "bluish discolouration" of the left fifth toe followed by similar changes in the right fifth toe. Two weeks before admission she had severe intermittent pain in both discoloured toes particularly at night. The pain was improved by walking and was partially relieved by analgesics. The pain was not exacerbated by exposure to cold. The patient did not report any symptoms of congestive heart failure including exertional dyspnoea, orthopnoea, paroxysmal nocturnal dyspnoea, and peripheral oedema. The patient had no history of arthritis or cerebrovascular disease. No history of rash, or symptoms attributable to a coagulation disorder, connective tissue disease, or vasculitis were elicited. There was nothing relevant in the patient's family history. The patient had consumed two ounces of vodka daily since the age of 18 and had smoked 40 cigarettes a day for 15 years.

\section{PHYSICAL EXAMINATION}

Examination showed: blood pressure 110/68 $\mathrm{mm} \mathrm{Hg}$, pulse 80 beats/min, respiration 16 breaths $/ \mathrm{min}$, and temperature $36 \cdot 5^{\circ} \mathrm{C}$. Fundoscopic examination did not show retinal haemorrhages, exudates, or other vascular abnormalities. Heart sounds were normal but there was a soft systolic ejection murmur along the left sternal border. The abdominal examination was entirely normal. The right and left fifth toes were cool, cyanotic, and exquisitely tender. Peripheral pulses were within normal limits. Both affected toes showed increased sensitivity to pin prick but the neurological examination was otherwise normal.

Blood chemistry values were normal. Complete blood count was normal except for a raised mean corpuscular volume of $112 \mathrm{fl}$. Prothrombin and partial thromboplastin times were normal. The erythrocyte sedimentation rate was $11 \mathrm{~mm} / \mathrm{h}$ (Westergren). Antinuclear antibody, rheumatoid factor, and cryoglobulins were not detected. Antithrombin 3, protein $\mathrm{C}$, and complement concentrations were normal.

The electrocardiogram showed $T$ wave inversion in the inferior and precordial leads. The chest $x$ ray showed slight enlargement of the cardiac silhouette. An aortogram and arteriogram of the toes were performed from the femoral approach. This showed occlusion of the digital arteries supplying the left fifth and third toes (figure). There was partial revascularisation from a collateral circulation. There was no angiographic evidence of atherosclerosis or vasculitis in the aorta or more 


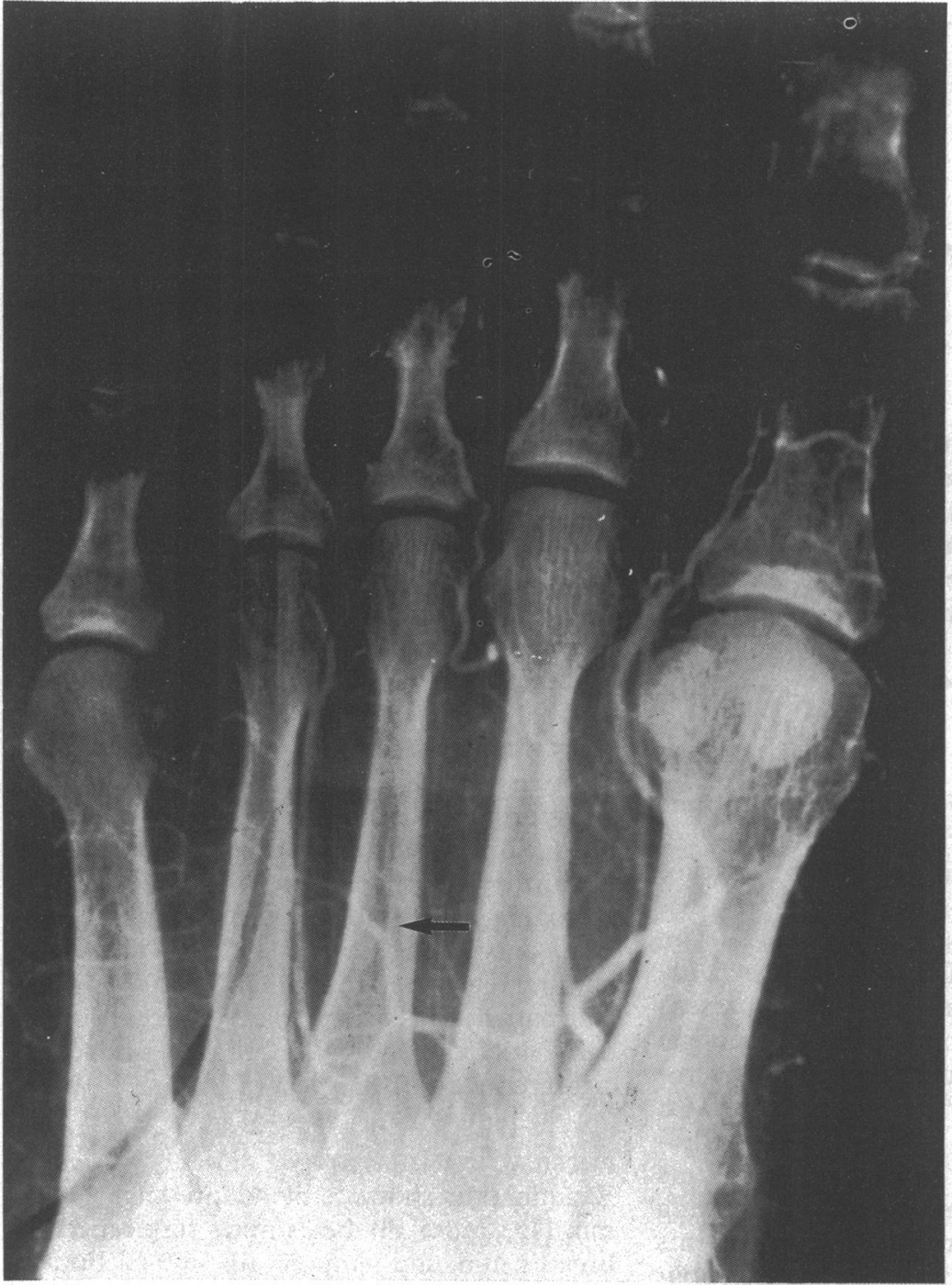

Arteriogram of the left foot showing complete occlusion of the digital arteries supplying the left fifth and third (arrow) toes.

distal vessels. $M$ mode and cross sectional echocardiography showed left ventricular enlargement, diffuse hypokinesis, and an apical mural thrombus. Radionuclide ventriculography showed a reduced left ventricular ejection fraction of $35 \%$, left ventricular enlargement, and global hypokinesis. Coronary angiography did not show luminal narrowing in the coronary arteries.

Because decreased left ventricular function could not be explained by coronary artery disease, right ventricular endomyocardial biopsy was performed to identify or rule out other myocardial abnormalities. Microscopy of the myocardial biopsy samples showed myofibre hypertrophy and increased interstitial fibrosis but no evidence of interstitial inflammatory infiltrates or myocyte necrosis consistent with the diagnosis of myocarditis. The small intramyocardial blood vessels seen in the endomyocardial biopsy samples were normal. The patient was treated with warfarin. The pain and cyanosis in the toes resolved within two months. The patient's cardiac function, monitored with serial physical examinations and radionuclide ventriculograms, improved progressively. A radionuclide ventriculogram performed nineteen months after the initial presentation showed a left ventricular ejection fraction of $42 \%$ and the disappearance of wall motion abnormalities. During the follow up period the patient did not drink any alcohol but she was unable to stop smoking.

\section{Discussion}

We report an unusual presentation of dilated cardiomyopathy in which systemic arterial microemboli preceded the symptoms and signs of congestive heart failure. Emboli to the lungs, kidneys, spleen, and brain are known to complicate the course of dilated cardiomyopathy and in some patients to precede all other cardiac symptoms. $^{45}$ Kyrle et al reported that peripheral embolisation complicates dilated cardiomyopathy. ${ }^{11}$ In this series, however, peripheral emboli occurred in patients with known cardiac dysfunction and were not the presenting sign that led to the diagnosis of dilated cardiomyopathy.

Ischaemia of the toes may be caused by peripheral vascular diseases including atherosclerosis, Buerger's disease, small vessel disease associated with diabetes mellitus, vasculitis, and microemboli. Angiography is helpful in identifying or ruling out these possibilities. In our case there were no angiographic changes of atherosclerosis, Buerger's disease, or vasculitis of large or medium sized arteries. Abrupt occlusion of the digital arteries was seen. This picture is compatible with embolisation, a vasculitis limited to the small arteries, or diabetic vascular disease. Vasculitis was believed to be an unlikely diagnosis because there were no constitutional symptoms to suggest a systemic inflammatory process. Nor did the laboratory data including a normal erythrocyte sedimentation rate, haemoglobin, and albumin, suggest an inflammatory vasculopathy. There were no other symptoms or signs to suggest a connective tissue disease and all serological testing was negative. The presence of diabetes mellitus was excluded by the absence of symptoms and retinal changes attributable to this disease and by the finding of a normal fasting blood glucose. By exclusion of other causes, embolisation was considered the most likely explanation for the digital artery occlusions.

The left ventricular mural thrombus seen on the cross sectional echocardiogram was the only identifiable source of the patient's microemboli.

The hypothesis that emboli originate from mural thrombi that form in the cardiac chambers as the result of blood stasis secondary to severely impaired systolic function is supported by the necropsy finding of cardiac thrombi in as many as $53 \%$ of patients with dilated cardiomyopathy. ${ }^{12}$ Other investigators have proposed that at least some embolic events in dilated cardiomyopathy are the manifestation of a generalised thrombotic tendency. This is suggested by a Japanese study of 36 patients with dilated cardiomyopathy. Four of these patients were young, suffered embolic events early in the course of their cardiac disease, which dominated the clinical picture, and died 
within a year of the onset of heart failure. ${ }^{13}$ Although a hypercoagulable state could not be conclusively ruled out in our patient, the normal platelet count and normal coagulation studies make the possibility of a generalised thrombotic tendency unlikely. Another possible cause of embolism in patients with dilated cardiomyopathy is arrhythmia. ${ }^{7}$ Our patient was in normal sinus rhythm and she had no electrocardiographic evidence of intermittent atrial fibrillation or complex ventricular arrhythmias.

Some investigators reported significant alterations in the activity of mitochondrial enzymes in the myocardial biopsy samples of patients with a history of excessive alcohol intake. ${ }^{14}$ On the basis of this finding it has been suggested that enzyme analysis of the biopsy samples may distinguish heart failure owing to alcohol from other causes of dilated cardiomyopathy. Biochemical analysis of the myocardial tissue was not performed in our patient. Three aspects of our patient's clinical picture, however, suggest that an unusual reaction to alcohol may have contributed to her cardiac dysfunction. These include a history of alcohol intake, the raised mean corpuscular volume, and the improvement of left ventricular function after she stopped drinking alcohol. Reversal of alcoholic cardiomyopathy has been previously reported by several investigators. ${ }^{15-17}$ Recent experimental evidence suggests that alcohol, by altering major regulatory mechanisms in the cell, has a direct toxic effect on the myocardium. ${ }^{1819}$ Thus it is possible that removal of the toxic insult improves cardiac function.

Another reversible cause of dilated cardiomyopathy is myocarditis. ${ }^{19}$ Myocardial inflammation was not seen on the patient's endomyocardial biopsy samples and she had no prodromes attributable to a viral infection. But because the diagnostic value of an endomyocardial biopsy specimen is limited by the possibility of sampling error, spontaneous resolution of myocarditis cannot be excluded in our patient. ${ }^{20}$

Peripheral microemboli originating from a cardiac mural thrombus can be the presenting symptom of dilated cardiomyopathy. Thus this disease entity should be identified or ruled out in patients showing evidence of peripheral microembolisation even when there are no symptoms of congestive heart failure.

1 Report of the WHO/ISFC task force on the definition and classification of cardiomyopathies. Br Heart $J$ 1980; 44:672-3.

2 Costanzo-Nordin MR. Anticoagulation and antiarrhythmic therapy in dilated cardiomyopathy. In: Engelmeier RS, therapy in dilated cardiomyopathy. In: Engelmeier RS, myopathy. New York: Marcel Dekker, 1988:71-68.

3 Gottdiener JS, Gay JA, Van Voorhees L, DiBianco R, Fletcher RD. Frequency and embolic potential of left ventricular thrombus in dilated cardiomyopathy: assessment of 2-dimensional echocardiography. Am J Cardiol 1983;52:1281-5.

4 Cosnett JE, Pudifin DJ. Embolic complications of cardiomyopathy. Br Heart $J$ 1964;64:544-8.

5 Parameswaran R, Meadows WR, Sharp JT. Coronary embolism in primary myocardial disease. Am Heart $J$ 1969;78:682-7.

6 Becker BJP, Chatgidakis CB, Van Lingen B. Cardiovascular collagenosis with parietal endocardial thrombosis: a clinical pathological report. Circulation 1953;7:345-56.

7 Fuster V, Gersh BJ, Giuliani ER, Tajik AJ, Brandenburg RO, Frye RL. The natural history of idiopathic dilated cardiomyopathy. Am J Cardiol 1981;47:525-30.

8 Lapeyre AC, Steele PM, Kazmier FJ, Chesebro JH, Vliestra RE, Fuster V. Low incidence of systemic embolism in left ventricular aneurysm-a comparison with idiopathic dilated cardiomyopathy. J Am Coll Cardiol 1983;1:704.

9 Johnson RA, Palacios I. Dilated cardiomyopathies of the adult. N Engl J Med 1982;307:1051-8, 1119-26.

10 Demakis JG, Rahimtoola SH, Sutton GC, et al. Natural course of peripartum cardiomyopathy. Circulation 1971; course of perip

11 Kyrle PA, Korninger C, Gossinger H, et al. Prevention of arterial and pulmonary embolism by oral anticoagulants in patients with dilated cardiomyopathy. Thromb Haemost patients with dilat

12 Roberts WC, Ferrans VJ. Pathological aspects of certain cardiomyopathies. Circulation 1974;34 (suppl II):128-44.

13 Koide T, Kato A, Takabatake Y, et al. Variable prognosis in congestive cardiomyopathy: role of left ventricular function, alcoholism and pulmonary thrombosis. Jpn Heart 1980;21:451-63.

14 Vikhert AM, Tsiplenkova VG, Cherpachenko, NH. Alcoholic cardiomyopathy and sudden cardiac death. Am Coll Cardiol 1986;8:3A-11A.

15 Demakis JG, Proskey A, Rahimtoola SH, et al. The natural course of alcoholic cardiomyopathy. Ann Intern Med 1974;80:293-7.

6 Regan T. Ethyl alcohol and the heart. Circulation 1971 44:957-63.

17 Alderman E, Coltart D. Alcohol and the heart. Br Med Bul 1982;38:77-80.

18 Urbano-Marquez A, Estruch R, Navarro-Lopez F, Grau JM, Mont L, Rubin E. The effects of alcoholism on skeletal and cardiac muscle. N Engl Med 1989;320:409-15.

19 Diamond I. Alcoholic myopathy and cardiomyopathy. $N$ Engl J Med 1989;320:458-60.

20 O'Connell JB, Constanzo-Nordin MR, Engelmeier RS, Wallis DE, Robinson JA, Scanlon PJ. Prognosis and treatment of cardiomyopathy and myocarditis. Heart and Vessels 1985; suppl I:175-9. 